\title{
Hydrogen Storage in a Potential Well for Room- Temperature Applications
}

\author{
Jaehyun Bae, Yea-lee Lee, Jeongwoon Hwang, Dongwook Kim, Jong Hyun Jung, Seungwook Son and Jisoon Ihm* \\ Department of Physics and Astronomy \\ Seoul National University \\ Seoul, 151-747, Republic of Korea \\ *Corresponding author
}

\begin{abstract}
Hydrogen has a gravimetric energy density nearly 3 times that of gasoline, and yet does not emit carbon dioxide at all in burning. However, since hydrogen exists in a bulky gas form at ambient conditions, the problem in the safe and efficient storage of hydrogen is a serious obstacle to the commercial use of hydrogen as a fuel. There is a recent proposal that, if we store the molecular hydrogen gas in a potential well created inside a material, the storage density increases by a Boltzmann factor which can be enormously large with a moderate potential depth even at room temperature. In the present study, we carry out a theoretical analysis for the storage at room temperature. Starting from a realistic description of hydrogen gas using the van der Waals equation of state, the expression for the density enhancement in the potential well is obtained. Especially, the first-order expansion of the equation provides us with an analytic solution which correctly shows the effects of the excluded volume and the dispersion interaction. We interpret and discuss the results taking into account the limitations of the van der Waals model.
\end{abstract}

Keywords-hydrogen storage; potential well; van der Waals equation

\section{INTRODUCTION}

Since the volumetric density of the molecular hydrogen gas is the lowest among all elements, the storage problem should be solved for the practical utilization of hydrogen [1]. One possible solution we may consider is to store hydrogen in a potential well, if some void space can be created inside materials with such an attractive potential for hydrogen gas. Suppose the molecular hydrogen gas experiences a potential energy $U(<0)$ inside a certain material and the outside free space has zero potential. Assuming that hydrogen is an ideal gas, the equilibrium condition between inside and outside the material ( $\mu_{\text {in }}=\mu_{\text {out }}$, where $\mu$ is the chemical potential of hydrogen molecules) gives us the necessary relation for the enhancement of density. The chemical potential $\mu$ of a free (without the potential) ideal gas at temperature $T$ is $k T \ln \left(\rho \lambda^{3}\right)$ [2] and that with potential $U$ is $k T \ln \left(\rho \lambda^{3}\right)+U$, where the density $\rho=N / V$, the thermal wavelength $\lambda=\left(h^{2} / 2 \pi M k T\right)^{1 / 2}, h$ is the Planck constant, $M$ is the mass of the $\mathrm{H}_{2}$ molecule, and $k$ is the Boltzmann constant. The relation between $\rho_{\text {in }}$ with the potential $U$ (inside the material) and $\rho_{\text {out }}$ without the potential (outside the material) is

$$
\rho_{\text {in }}=\rho_{\text {out }} \mathrm{e}^{-U / k T} .
$$

This relation is the basis for the huge density enhancement we are going to investigate below; with a relatively small potential depth $|U|=0.12 \mathrm{eV}$, the Boltzmann factor is as large as 100 at room temperature ( $T=300 \mathrm{~K})$.

\section{STORAGE ENHANCEMENT FOR REALISTIC $\mathrm{H}_{2}$ GAS}

For a reasonably safe handling of compressed $\mathrm{H}_{2}$, a generally accepted upper limit of the pressure is $100 \mathrm{~atm}$ which is also the target value recommended by the United States Department of Energy by the year 2017 [3]. Since the enhancement factor is large for a moderate potential well at room temperature, the $\mathrm{H}_{2}$ gas inside the storage material reaches a very high density state at a relatively low outside pressure (say, $10 \mathrm{~atm}$ ). Therefore, the ideal gas law is almost always broken in practical storage situations and we require a more realistic description of $\mathrm{H}_{2}$ gas confined inside the potential well including the effects of the so-called dispersion (or van der Waals) interactions as well as the repulsive cores. We choose the van der Waals equation of state [2] for such a purpose,

$$
\left(P+N^{2} a / V^{2}\right)(V-N b)=N k T,
$$

where $N$ is the total number of $\mathrm{H}_{2}$ molecules, $a=6.764 \times 10^{-50} \mathrm{~Pa} \cdot \mathrm{m}^{6}$ and $b=4.402 \times 10^{-29} \mathrm{~m}^{3}$ for $\mathrm{H}_{2}$ [4]. For the free $(U=0)$ van der Waals gas, $\mu$ is known to be $k T\left\{\ln \left[\rho \lambda^{3} /(1-\rho b)\right]+\rho b /(1-\rho b)\right\}-2 \rho a[2]$. In this case, the equilibrium condition between inside and outside gives,

$$
k T\left(\ln \frac{\rho_{\text {in }} \lambda^{3}}{1-\rho_{\text {in }} b}+\frac{\rho_{\text {in }} b}{1-\rho_{\text {in }} b}\right)-2 \rho_{\text {in }} a+U
$$




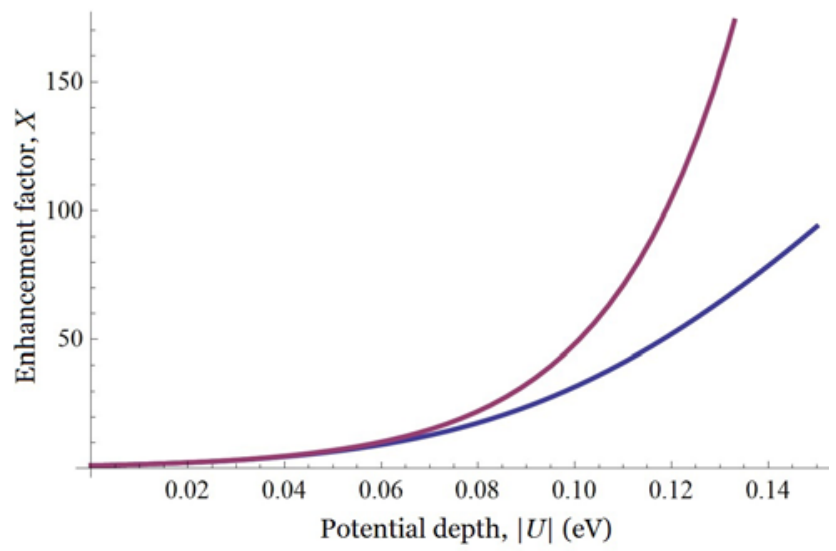

FIGURE I. DENSITY ENHANCEMENT FACTOR AS A FUNCTION OF THE POTENTIAL DEPTH $|U|$ at $P_{\text {out }}=10$ ATM AND $T=300 \mathrm{~K}$. THE PURPLE LINE INDICATES THE IDEAL GAS, AND THE BLUE LINE DENOTES THE VAN DER WAALS GAS FROM THE LINEARIZED EQUATION (4) IN THE TEXT.

$$
=k T\left(\ln \frac{\rho_{\text {out }} \lambda^{3}}{1-\rho_{\text {out }} b}+\frac{\rho_{\text {out }} b}{1-\rho_{\text {out }} b}\right)-2 \rho_{\text {out }} a
$$

Although the equation can in principle be solved numerically, it is very instructive to make an assumption that $\rho_{\mathrm{in}} b$ is smaller than 1 and to keep the leading term in the expansion in $\rho_{\text {in }} b$ in order to obtain an analytic solution. (It is also implicitly assumed that the gas does not make a phase transition to a liquid phase, which is always the case around room temperature.) Then we have,

$$
\begin{aligned}
& k T \ln \left(\rho_{\text {in }} \lambda^{3}\right)+2 \rho_{\text {in }} k T\left(b-\frac{a}{k T}\right)+U \\
& =k T \ln \left(\rho_{\text {out }} \lambda^{3}\right)+2 \rho_{\text {out }} k T\left(b-\frac{a}{k T}\right) .
\end{aligned}
$$

We finally obtain the equation for the volumetric number density enhancement factor $X \equiv \rho_{\text {in }} / \rho_{\text {out }}$,

$$
\ln X+2 \rho_{\text {out }} b\left(1-\frac{u_{0}}{T}\right)(X-1)+\frac{U}{k T}=0
$$

where $u_{0} \equiv a / b$ is known to be the magnitude of the effective van der Waals potential $(=9.6 \mathrm{meV})$. Sometimes $b$ is written in terms of the effective diameter $(D)$ of the van der Waals excluded volume, namely, $b=\frac{2}{3} \pi D^{3}$. Equation (5) has an

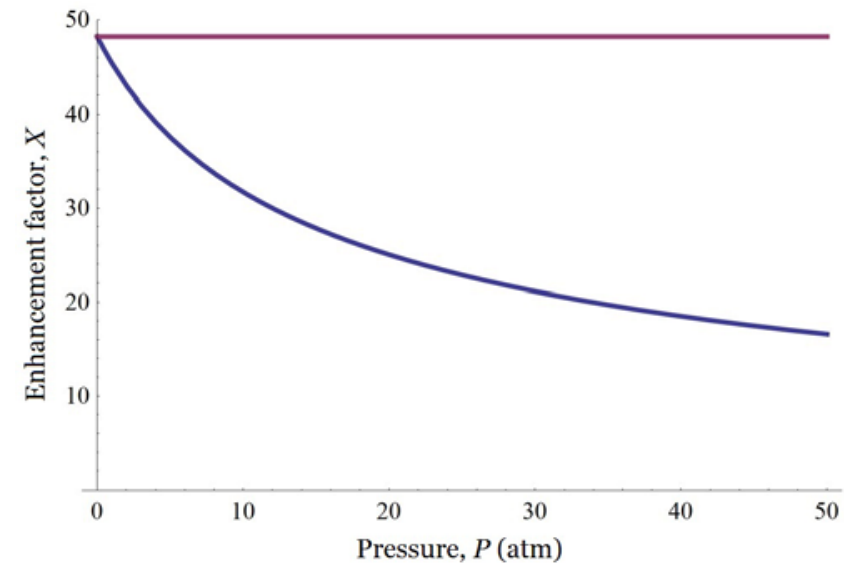

FIGURE II. DENSITY ENHANCEMENT FACTOR AS A FUNCTION OF PRESSURE AT $U=-0.1 \mathrm{eV}$ AND $T=300 \mathrm{~K}$. THE PURPLE LINE INDICATES THE IDEAL GAS, AND THE BLUE LINE DENOTES THE VAN DER WAALS GAS FROM THE LINEARIZED EQUATION (4) IN THE TEXT.

analytic solution known as the "Product Log" ( PL ) function [5]. $\operatorname{PL}(z)$ is defined by the principal solution in the equation $\mathrm{z}=\mathrm{PL}(\mathrm{z}) \mathrm{e}^{\mathrm{PL}(z)}$ for a complex number $z$ in general. In our case, both $z$ and $\operatorname{PL}(z)$ are real. Now the solution to (5) is

$$
X(U, P, T)=\frac{1}{A} \operatorname{PL}[A \exp (A-U / k T)],
$$

where $A=2 b\left(1-u_{0} / k T\right) \rho_{\text {out }}$ and $\rho_{\text {out }}$ is of course a function of $P$ and $T$. Equation (6) reflects a significant modification to a simple expression for the density enhancement of the 3D ideal gas (Equation (1)).

\section{RESULTS AND DISCUSSION}

The results for the $\mathrm{H}_{2}$ density enhancement factor $X$ in (6) are plotted in two different ways. For a low applied pressure of $10 \mathrm{~atm}$ at room temperature (300K), the enhancement factor is plotted as a function of the potential depth $|U|$ in Fig. 1. The behavior expected for an ideal gas is presented as well for comparison. For small $|U|$, the curve follows the exponential Boltzmann factor of $\exp (|U| / k T)$ as expected. When $|U|$ increases further, the slope of the curve tends to be reduced relative to the ideal gas, indicating that the non-ideal behavior (the excluded volume and the $\mathrm{H}_{2}-\mathrm{H}_{2}$ dispersion interaction) becomes appreciable. In Fig. 2, the enhancement factor is plotted as a function of the applied pressure at $T=300 \mathrm{~K}$ for a given potential energy $U=-0.1 \mathrm{eV}$. The behavior of an ideal gas is again presented for comparison. At a very low pressure close to $1 \mathrm{~atm}$, it is practically identical to $\exp (-U / k T)$. For increased $P$, the enhancement factor decreases substantially as expected for a realistic gas. Also, it is to be reminded that as 
$|U|$ or $P$ increases much more, not only the linearized equation (4) but also the original van der Waals gas relation (3) becomes inaccurate in describing the actual $\mathrm{H}_{2}$ gas.

In including the effects of the $\mathrm{H}_{2}-\mathrm{H}_{2}$ interaction in Section II, we have employed the parameters obtained by fitting to the experimental critical point of $\mathrm{H}_{2}$ within the van der Waals model [4]. Actually, we have performed first-principles density functional theory (DFT) calculations $[6,7]$ using the generalized gradient approximation of Perdew et al. [8] with the Vienna $a b$ initio simulation package [9] including the Grimme type van der Waals interaction corrections [10] to obtain the $\mathrm{H}_{2}-\mathrm{H}_{2}$ interaction. Another interaction potential proposed by Silvera et al. [11] is widely adopted in the field. (The well-known Lennard-Jones potential [12] is also a possible choice to take into account the interaction.) The Silvera-Gold potentials produce a less attractive potential and our DFT calculations produce a more attractive potential than the abovementioned values fitted to the experimental critical point. Still, we note that the dramatic increase in the storage density by the potential well remains to be valid irrespective of the details of the $\mathrm{H}_{2}-\mathrm{H}_{2}$ interaction parameters.

In conclusion, we have shown that a potential well produced in the hydrogen storage material can increase the storage capacity remarkably, and the behavior of the storage density enhancement in a realistic situation may be understood either numerically or analytically with a good precision using the model examined here. We anticipate that the proposed mechanism to enhance the hydrogen storage using the potential well will contribute greatly to the development of the roomtemperature hydrogen storage materials.

\section{ACKNOWLEDGMENT}

This work was supported by the NRF funded by the MSIP of the Korean government (Grant No. 2006-0093853). Computations were supported by the Korea Institute of Science and Technology Information.

\section{REFERENCES}

[1] L. Schlapbach, and A. Zuttel, "Hydrogen-storage materials for mobile applications,” Nature, vol. 414, pp. 353-358, 2001.

[2] H. Koemer, and C. Kittel, Thermal Physics. WH Freeman, 1980.

[3] M. P. Suh, H. J. Park, T. K. Prasad, and D.-W. Lim, "Hydrogen Storage in Metal-Organic Frameworks,” Chem. Rev. vol. 112, pp. 782-835, 2012.

[4] D. R. Lide, CRC Handbook of Chemistry and Physics. Boca Raton, FL: CRC Press, p. 6-48, 1994.

[5] R. M. Corless, G. H. Gonnet, D. E. Hare, D. J. Jeffery, and D. E. Knuth, "On the LambertW function,” Adv. Comput. Math. vol. 5, pp. 329-359, 1996.

[6] P. Hohenberg, and W. Kohn, "Inhomogeneous electron gas," Phys. Rev. vol. 136, pp. B864-B871, 1964.

[7] W. Kohn, and L. J. Sham, "Self-consistent equations including exchange and correlation effects,” Phys. Rev. vol. 140, pp. A1133-A1138, 1965.

[8] J. P. Perdew, K. Burke, and M. Ernzerhof, "Generalized gradient approximation made simple,” Phys. Rev. Lett. vol. 77, pp. 3865-3868, 1996.

[9] G. Kresse, and \& J. Furthmüller, "Efficient iterative schemes for $a b$ initio total-energy calculations using a plane-wave basis set," Phys. Rev. B, vol. 54, pp. 11169-11186, 1996.

[10] S. Grimme, "Semiempirical GGA-type density functional constructed with a long-range dispersion correction,” J. Comput. Chem. vol. 27, pp. 1787-1799, 2006.

[11] I. F. Silvera, and V. V. Goldman, "The isotropic intermolecular potential for $\mathrm{H}_{2}$ and $\mathrm{D}_{2}$ in the solid and gas phases," J. Chem. Phys. vol. 69, pp. 4209-4213, 1978.

[12] J. E. Lennard-Jones, “On the determination of molecular fields,” Proc. R. Soc. London, Ser. A, vol. 106, pp. 463-477, 1924. 